\title{
La enseñanza en vilo de las emociones: una perspectiva emocional de la educación
}

\section{John Fredy Henao-Arias}

orcid.org/00oo-0002-8840-1647

Universidad Católica de Manizales,

Colombia

johenaoa@unal.edu.co
José Hoover Vanegas-García

orcid.org/0ooo-0002-1424-7990

Universidad Autónoma de Manizales,

Colombia

hovg@autonoma.edu.co

\section{Andrés Elías Marín-Rodríguez}

orcid.org/o0oo-0002-2279-1419

Universidad Católica de Manizales,

Colombia

\section{Resumen}

El artículo tiene como objetivo principal la revisión de artículos de revistas indexadas, tesis, libros y capitulos de libros que han tratado la cuestión de las emociones, pero más especificamente el tema de las emociones en la enseñanza. Teniendo en cuenta las investigaciones más importantes que se han realizado a nivel mundial desde 1993 hasta el 2016. Se presenta una aproximación al término "emoción", desde referentes teóricos contemporáneos y desde diferentes campos de conocimiento, como la neurociencia, la psicología, la sociología y la filosofía; luego se examina el trabajo emocional y la geografía emocional de la enseñanza. La conclusión resalta que las emociones juegan un papel primordial en el proceso de enseñanza, pues la disposición del maestro para enseñar depende de ellas; además, son mediadores en la toma de decisiones de los maestros en los actos pedagógicos. Este trabajo pertenece al proyecto de investigación "La ira, la alegría y la tristeza de los profesores: una mirada desde la enseñanza", en el marco de la maestría de la Maestría de Educación, de la Universidad Católica de Manizales.

\section{Palabras clave}

Emociones; educación; enseñanza; estados de ánimo; interacciones (Fuente: Tesauro de la Unesco).

Recepción: 20-09-2016 | Envío a pares: 18-05-2017 | Aceptación por pares: 15-06-2017 | Aprobación: 14-07-2017 


\title{
Education Hinging on Emotions: An Emotional View of Education
}

\begin{abstract}
The main objective of this article is to review articles from indexed journals, dissertations, books and book chapters that deal with the issue of emotions, but more specifically, the topic of emotions in teaching. It takes into account the most important studies that were conducted worldwide from 1993 to 2016. An approach to the term "emotion" is presented based on contemporary theoretical referents and different fields of knowledge, such as neuroscience, psychology, sociology and philosophy. Then, the emotional work and the emotional geography of teaching are examined. The conclusion emphasizes that emotions play a key role in the teaching process, since the teacher's willingness to instruct depends on them. Moreover, emotions are mediators in the teacher's decision-making with respect to educational action. This work is part of "La ira, la alegría y la tristeza de los profesores: una mirada desde la enseñanza" ("Teachers' Anger, Joy and Sadness: Explored from the Standpoint of Teaching"), a study developed for a master's degree in education at the Universidad Católica de Manizales.
\end{abstract}

Keywords

Emotions; education; teaching; moods; interactions (Source: Unesco Thesaurus). 


\title{
O ensino à beira das emoções: uma perspectiva emocional da educação
}

\begin{abstract}
Resumo
Este artigo tem como objetivo principal revisar artigos de revistas indexadas, teses, livros e capítulos de livros que tratam da questão das emoções, mas, em especial, do tema das emoções no ensino. Para isso, consideram-se as pesquisas mais importantes realizadas em todo o mundo desde 1993 até 2016. Apresenta-se uma aproximação ao termo emoção a partir de referenciais teóricos contemporâneos e de diferentes campos do conhecimento, como a neurociência, a psicologia, a sociologia e a filosofia; em seguida, examinam-se o trabalho emocional e a geografia emocional do ensino. A conclusão ressalta que as emoções desempenham um papel primordial no processo de ensino, pois a disposição do professor para ensinar depende delas; além disso, são mediadoras na tomada de decisões dos professores nas ações pedagógicas.
\end{abstract}

\section{Palavras-chave}

Educação; emoções; ensino; humor; interações (Fonte: Tesauro da Unesco). 


\section{Delimitación del tema}

Los actos pedagógicos o relaciones en la formación siempre están acompañados de interacciones y entre ellas la comunicación es el eje o la urdimbre que permite que los seres humanos se entiendan, se pongan de acuerdo, para que no se impongan los unos sobre los otros. En este escenario no solo entran en escena las palabras o los lenguajes corporales, pues también cuentan las emociones, esos estados anímicos que acompañan a todos los seres humanos, en todas las circunstancias, tanto en la vida cotidiana como en la vida reflexiva. Las emociones, como los pensamientos, los sentimientos, los valores y las creencias son dispositivos que los seres humanos experimentan en todo momento; sin embargo, si hay un dispositivo que genere afección directa en las personas son las emociones, pues ellas las habitan no solo como proceso neurológico, sino como factor mediador en la comunicación y como elemento fundamental en la toma de decisiones.

De estas consideraciones nace la necesidad de indagar por el papel de las emociones en los actos de enseñanza dentro del aula de clase por medio de una investigación comprensiva, en un intento de visualizar la educación desde una concepción humanista y no al modo de la reflexión tradicional en donde se excluye a las emociones, como lo afirma Gardner: "bajo la égida del positivismo, sólo se aceptaban los hechos materiales sólidos y no tenían cabida las ideas, las emociones ni los valores" (1997, p. 70). Esta investigación pretende dar cuenta de las incidencias más predominantes de los maestros en sus actividades de enseñar, pero para ello es necesario hacer un recorrido, primero, por la literatura que da cuenta de las emociones y, segundo, por la relación entre estas y la educación, sobre lo cual se encuentran varios documentos que tratan el tema con rigurosidad. De esta manera, a continuación presentamos la revisión teórica que se elaboró como punto de partida para la realización de la investigación.

Para identificar las fuentes primarias y secundarias se usaron diferentes referencias bibliográfi- cas entre marzo del 2015 y agosto del 2016, hemerotecas virtuales como Scielo y Redaly c, al igual que repositorios digitales, pero principalmente se hizo un rastreo bibliográfico a través de Google Academics combinando los descriptores: "emoción", "enseñanza”, "docente", "ambiente de aula”, "emotion”, "teaching and teacher". Asimismo, artículos de revistas indexadas, tesis, libros y capítulos de libros sirvieron de referencia en la indagación de nuevas fuentes teóricas y metodológicas que alimentaron posteriores búsquedas en bases de datos. De esta manera, se logró tener la mayor cantidad posible de fuentes para realizar una revisión rigurosa. Luego de esto, se seleccionó la bibliografía que abordara de forma concreta las categorías enseñanza, emociones, profesores y respondiera a los objetivos propuestos para el presente artículo.

\section{Aproximación al término emoción}

En la actualidad, es usual escuchar en diversos espacios académicos y no académicos el término emoción y, de hecho, desde los años 90 se ha convertido en un tema común en el área de la educación. Estudiantes, docentes y directivos tienen el término incorporado a sus campos enunciativos; sin embargo, cuando se interpela por una definición concreta, las respuestas se hacen ambiguas y oscuras.

Al ser las emociones una realidad común, su análisis se repliega a la cotidianidad y, por consiguiente, no se hace una distinción concienzuda de las mismas. Precisamente por ello, cuando se indaga por una conceptualización del término, se acude a hacer un listado de las emociones más frecuentes, como la ira, la alegría, la tristeza, el miedo, entre otras. Tenemos, pues, que, a pesar de que todos los seres sintientes viven atravesados por las emociones, la definición del concepto se hace escurridiza. El diccionario de la Real Academia Española define emoción (del latín emotio, ōnis) como "alteración del ánimo intensa y pasajera, agradable o penosa, que va acompañada de cierta conmoción somática". Aquí se tiene una primera aproximación; sin embar- 
go, esta definición se queda corta ante la profundidad del fenómeno emocional, ya que, si se entra a hacer un análisis detenido de este, nos encontraremos con una gran variedad de abordajes teóricos del fenómeno, que hay que diferenciar, pues en ocasiones resultan contradictorios.

Para empezar, es claro que desde la Antigüedad y a lo largo de la historia se ha hablado de las emociones y que, por tanto, no es un tema nuevo; no obstante, estas fueron relegadas desde el siglo XVII por la tradición basada en los postulados cartesianos a un análisis dualista de la mente y del cuerpo, atribuyendo en algunos casos las emociones al cuerpo y en otros a la mente. Como lo señalan Colhoun y Solomon, "Descartes no se limita a un análisis fisiológico de la emoción. También describe las emociones en lenguaje mentalístico directo, y habla de las percepciones, los deseos y las creencias relacionadas con las diferentes emociones" (1996, p. 62). De aquí se desprenden dos grandes corrientes interpretativas en el estudio de las emociones. Por un lado, tenemos la teoría de la sensación, que edificaría sus argumentos a partir de los fenómenos fisiológicos de la emoción o, dicho de otra manera, en sus manifestaciones corpóreas. Esta corriente fue dominante hasta el siglo XIX. Por otro lado, tenemos las teorías cognitivistas que toman como fundamento las creencias en las que se fundan las emociones, es decir, enfocadas en el componente mental. Esta línea es seguida principalmente por la filosofía analítica. En el presente texto se abordarán las principales teorías contemporáneas, más exactamente las que se han dado desde 1883 hasta la fecha.

Así pues, uno de los primeros autores contemporáneos en tratar de responder la pregunta por la emoción, con un enfoque científico, fue el filósofo y psicólogo William James (1842-1910) a través de su ensayo What is an emotion? En él presenta la hipótesis de que "los cambios corporales son posteriores a la percepción de un hecho desencadenante y que nuestra sensación de esos cambios según se van siguiendo es la emoción" (1884, pp. 189-190). En este sentido, el único escenario de las emociones es el cuerpo y no habría ningún componente mental, pues el autor norteamericano sigue disertando en coherencia con la heredada postura cartesiana de base fisiológica y usa un ejercicio mental para persuadir a sus lectores: "si tratamos de abstraer de nuestra conciencia de la emoción todas las sensaciones de sus sintomas corporales característicos, no quedaría nada atrás, ningún componente mental" (p. 193). Es decir que, si al sentir ira se abstraen sus componentes fisiológicos, como el aumento de la presión sanguínea, del ritmo cardiaco y de la adrenalina, la ira desaparece inmediatamente con ellos. Visto de esta forma, las emociones no serían cognitivas. Esta hipótesis es conocida como la teoría JamesLange, pues el psicólogo danés Carl Lange (18341900) quien hizo hallazgos similares (1967, p. 66) a los de James, a pesar de trabajar de forma separada. Esta hipótesis reduce las emociones a la percepción de cambios fisiológicos luego de un estímulo externo. Desde esta perspectiva, las emociones son la conciencia de los cambios corporales, de lo que se deduce que las emociones solo pueden ser conscientes. Sobre esta concepción de las emociones se fundan todas las teorías contemporáneas no cognitivas.

Al respecto, Sigmund Freud (1856-1939) afirmaba, siguiendo la teoría James-Lange, que "la respuesta a la pregunta por las sensaciones, los sentimientos, los afectos inconscientes se resolvería con igual facilidad. Es que el hecho de que un sentimiento sea sentido y, por lo tanto, que la conciencia tenga noticia de él es inherente a su esencia" (1916, p. 173). Sin embargo, para demostrar que también existen sentimientos inconscientes, argumentaba que, a pesar de que el afecto es percibido conscientemente, la interpretación que hacemos de este, en ocasiones, no es ajustada, pues está enlazado a una idea errónea que es considerada por la conciencia como aquello que causa el afecto. En este sentido, el sentimiento sería inconsciente, mas no su afecto.

Como ya se enunció anteriormente, esta forma de interpretar el fenómeno de las emociones fue el 
paradigma predominante hasta la aparición de las teorías evaluativas y cognitivas, a partir de la segunda mitad del siglo XX. Según Vendrell, "esto se dio por la definición de la conciencia como intencional por parte de Franz Brentano (1838-1917) y por el interés en la función adaptativa al entorno que cumple la conciencia" (2009, p. 227). En otras palabras, la conciencia dejó de estar ensimismada y pasó a estar arrojada a los objetos que están en el mundo; en consecuencia, las nuevas teorías sobre las emociones dejaron de lado al cuerpo como el objeto de estas para ponerlo en las situaciones, personas o cosas hacia las cuales se dirige la intención de aquel que siente la emoción. De lo anterior se desprende una nueva forma de entender el fenómeno de las emociones en donde nuevos elementos entraban a ser considerados por los teóricos del campo, tales como las creencias, los juicios, la intencionalidad y la evaluación.

Por ejemplo, Victoria Camps advierte que "aunque la afección de entrada es corporal y está provocada por algo externo a nosotros, en el fondo de ella yace algún pensamiento o creencia relativo a lo que acabamos de percibir, y que nos lo señala como algo temible o digno de atención" (2011, p. 28). Vistas desde esta perspectiva, las creencias son fundamentales para que la emoción aparezca. En este sentido, la emoción no es algo que simplemente afecte al ser humano, algo que le sobreviene sin que este pueda hacer nada para controlarla, ya que, al estar basada en una creencia, el ser humano hace que la emoción sea. En otras palabras, si con James-Lange la emoción desaparecía, al abstraer todos sus efectos corpóreos, con Camps la emoción desaparece, si desaparece la creencia que la alimenta; por consiguiente, "todos los sentimientos se explican por conocimientos o [por las] creencias que los sustentan" (p. 28).

Adscrita también a esta línea cognitivista, tenemos a la filósofa estadounidense Martha Nussbaum quien defiende la hipótesis de que "las emociones comportan juicios relativos a cosas importantes, evaluaciones en las que, atribuyendo a un objeto externo relevancia para nuestro bienestar, recono- cemos nuestra naturaleza necesitada e incompleta frente a porciones del mundo que no controlamos plenamente" (2001, p. 41). Esta postura no es nueva, pues se despliega desde el postulado estoico, aunque niega de forma vehemente la idea de que las emociones deban ser extirpadas de la vida práctica de los seres humanos, con el fin de alcanzar el bienestar; es decir, en coherencia con los estoicos, que las emociones son un obstáculo que desde la irracionalidad turba el ánimo humano y empuja su voluntad para realizar acciones que en ocasiones resultan incomprensibles en toda lógica.

Concordando con la postura de Nussbaum, el neurocientífico A. Damasio (1994) aduce que las emociones, aparte del componente corpóreo, tienen un componente cognitivo importante; de hecho, Damasio une ambos componentes al proponer la hipótesis del marcador somático, la cual apunta a clarificar el proceso de toma de decisiones que, muy al contrario de lo que se creería, no está basado en un cálculo de costo-beneficio con el fin de recibir el mayor bien posible, sino que es una respuesta automática en el cuerpo, que reduce las posibilidades en la toma de decisiones. La toma de decisiones depende de las señales que las respuestas biorreguladoras envían, respuestas que apuntan a mantener la homeostasis y asegurar la supervivencia (Gazzaniga, 1999, p. 1047). En palabras de Damasio, "los marcadores somáticos son un caso especial de sentimientos generados a partir de emociones secundarias que han sido conectados, mediante aprendizaje, a resultados futuros predecibles de determinados supuestos" (1994, p. 200).

En otras palabras, cuando estamos frente a una situación en la cual hay que tomar una decisión, los marcadores somáticos reducen el rango de elección y desechan las opciones que generan emociones negativas; por ejemplo, si me encuentro en una situación en la cual se repiten las circunstancias de un momento que me generó tristeza en el pasado, esta proyecta su sombra al futuro en el momento que estoy tomando la decisión y, por tanto, excluyo 
esta opción. Al respecto, Gazzaniga afirma que "no importa cuántas ideas racionales una persona sea capaz de idear, la emoción es necesaria para tomar decisiones" (2008, p. 120).

De esta forma, Damasio hiere de muerte la idea de que las emociones son la antítesis de la mente científica, y lo hace desde la misma ciencia, pues su teoría está fundada en estudios neurológicos con personas que sufrieron lesiones cerebrales. Principalmente se basa en el caso de Phineas Gage, un capataz de construcción que sufrió un accidente en el que una barra metálica atravesó su cráneo, cuya personalidad, tras recuperarse, cambió, al punto que, a pesar de razonar de manera coherente, era incapaz de tomar decisiones, no daba el pasó final, a causa de que las áreas del cerebro en las que convergían las emociones y la toma de decisiones se vieron seriamente afectadas tras el accidente. En consecuencia, se hace necesario repensar la relación que existe entre las emociones y la razón, tanto en la vida cotidiana como en el campo científico, pues, a pesar de que las emociones no funcionan con la lógica científica, sí son necesarias para tomar las decisiones que crean ciencia. En este sentido, la emoción es indispensable para la razón y, a su vez, la razón alimenta las emociones guisada por las creencias; en otras palabras, hay una relación directa entre la una y la otra que las hace interdependientes.

Al proponer la teoría de los marcadores somáticos, Damasio abandona la dualidad fundada por Descartes, une la emoción (cuerpo) con la cognición (mente) y, además, pone en ellas un nuevo aspecto: el aprendizaje. Puesto que los marcadores somáticos tienen que ver con experiencias vividas que quedaron ancladas, "es probable que la mayoría de marcadores somáticos que utilizamos en la toma racional de decisiones se haya creado en el cerebro en el proceso de educación y socialización [...]. En otras palabras, se basan en el proceso de emociones secundarias" (1994, p. 203).

Al respecto, cabe aclarar que se han realizado diversas taxonomías de las emociones, pero estas di- vergen entre sí. Entre las más populares tenemos las de Ekman $(1973,1993,1998)$ y Ekman y Oster (1981), las cuales -en la línea no cognitivista, y siguiendo los estudios de Charles Darwin (1809-1882) con tribus indígenas en Papúa Nueva Guinea- llegaron a la conclusión de que hay unas emociones básicas o primarias, pues se repiten alrededor del mundo y son fácilmente identificables por cualquier persona. Estas emociones serían: alegría, ira, miedo, asco, sorpresa, tristeza (Ekman y Oster, 1981, p. 120). Por su parte, Plutchik (1980) propuso una rueda cromática de ocho emociones primarias, a saber: alegría, tristeza, ira, miedo, sorpresa, disgusto, aceptación y anticipación. También influido por Ch. Darwin, Plutchik postula que estas emociones primarias están encaminadas a la supervivencia de las especies e indica que su combinación por parejas produce emociones secundarias, con los siguientes resultados: alegría + confianza = amor; confianza + miedo = sumisión; tristeza + ira = envidia; disgusto + tristeza $=$ remordimiento (citado en Díaz y Flores, 2001, p. 21).

Retomando a Damasio, existen dos tipos de emociones: las primarias (tempranas) que son innatas, preorganizadas, jamesianas, en las cuales juega un papel primordial el sistema límbico, y las emociones secundarias (adultas), las cuales, además de dispararse en el sistema límbico, usan las capas corticales prefrontales y somatosensoriales y surgen de la evocación de imágenes verbales o no verbales, es decir, que pensamos en palabras y en imágenes (1994, pp. 155-160). En esto difiere de la teoría de Nussbaum, quien, como ya se enunció anteriormente, parte de juicios de valor eminentemente verbales, es decir, lingüísticos.

No obstante, cabe anotar una importante diferencia en la teorización de las emociones entre Damasio y Nussbaum, ya que ambos hablan de bienestar desde un punto de vista evaluativo, pero desde perspectivas totalmente diferentes. Para el neurocientífico, el bienestar tiene que ver con la homeostasis, que se puede definir como un aprovisionamiento connatural y automatizado de la ad- 
ministración de la vida y su equilibrio. En el caso de las emociones, estas se encuentran en la cúspide del esquema homeostático y su objetivo sería "proporcionar un estado vital mejor que neutro, que como animales pensantes y ricos identificamos como comodidad y bienestar" (Damasio, 2005, p. 39). Por el lado de la filósofa, el bienestar tiene que ver con el término

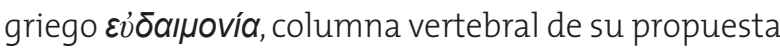
teórica. En ella, la eudemonía -en su sentido más amplio, y evitando la traducción de felicidad- se concibe como florecimiento humano, entendido como vida plena, en la cual poseemos todas las cosas a las que atribuimos valor, dentro de nuestro esquema de fines y objetivos (Nussbaum, 2001, pp. 53-54). Tenemos entonces que tanto Damasio como Nussbaum, en sus constructos teóricos sobre las emociones, hablan de evaluación, pero el primero lo hace desde la postura evolucionista, por lo que para él la evaluación busca determinar las amenazas externas, mientras en Nussbaum la evaluación tiene que ver con lo que se considera importante para cumplir con el esquema de objetivos, lo cual implica que está orientada al futuro.

\section{El trabajo emocional de la enseñanza}

Como el trabajo del profesor no es solo una labor física y mental, sino altamente emocional (Ye y Chen, 2015), según Fried "se hace indispensable un mayor entendimiento del papel de las emociones en la profesión docente, pues esto puede ayudar en la formación de maestros que estén bien equipados para hacer frente a las demandas de la clase" (2011, p. 7). En este terreno, se ha integrado a la investigación educativa la teoría del Trabajo Emocional, la cual usa la terminología propia de la economía para analizar el papel de las emociones en el sector terciario o de servicios, dentro del cual se encuentra la educación. Visto desde esta perspectiva, el docente es un empleado y los estudiantes y sus padres son sus clientes. La propuesta del trabajo emocional, a su vez, está basada en los trabajos de Erving Goffman (1922-1982), padre de la microsociología, quien advertía que en la vida cotidiana las personas juegan diferentes roles en diferentes escenarios, para crear impresiones que los favorezcan (1981).

A. R. Hochschild traslada el trabajo de Goffman al campo de las emociones para proponer que las personas escenifican (perform) sus emociones de acuerdo con el contexto. En este sentido, define el trabajo emocional como "la gestión de la emoción con el propósito de crear una semblanza facial y corporal observable públicamente con el fin de obtener un salario" (1983, p. 7). De esta suerte, como las emociones son una mercancía que se intercambia, el empleado demuestra las adecuadas para que el cliente se sienta cómodo y contento con el trabajo que este desempeña, pues es lo que le conviene a la empresa y lo que la empresa exige. Ashforth y Humphrey sostienen que el trabajo emocional aparece cuando "el trabajador intenta dirigir su comportamiento hacia los demás con el fin de fomentar tanto percepciones sociales para sí mismo o sí misma como un cierto clima interpersonal favorable" (1993, p. 90). Ambas definiciones están enfocadas en la conducta del empleado. Por su parte, Grandey defiende que el trabajo emocional está encaminado a satisfacer las expectativas de la organización y hace énfasis en un aspecto más psicológico, pues lo define como "el proceso de regular los sentimientos y las expresiones para alcanzar las metas organizacionales" (2000, p. 97). Ambas definiciones son complementarias, pues en el trabajo emocional la conducta observable va acompañada de emociones internas que la sustentan (Gracia, Ramos, y Moliner, 2014).

Hochschild (1979, 1983, 1990) encuentra tres tipos de gestión emocional, a saber: acción superficial, acción profunda y expresión natural de los sentimientos. La primera se asocia a las situaciones en las cuales el individuo no cumple con los requerimientos de la empresa, así que el empleado cambia la expresión para cambiar las emociones; en otras palabras, se simula la emoción en su manifestación corpórea, pero se la cambia. Por contraste, en la segunda variedad de gestión emocional, el individuo 
cambia las emociones para cambiar las expresiones y se asocia a las situaciones en las cuales el empleado altera sus sentimientos para alinearlos con las expectativas organizacionales, con lo que tiene expresiones más naturales y genuinas (Grandy, Diefendorff y Rupp, 2013; Morris y Feldman, 1996). Finalmente, la expresión natural de las emociones se refiere a la consistencia de las emociones que refleja el empleado, entre lo que siente internamente y lo que la organización requiere.

Asimismo, Hochschild (1983) aclara que los empleos que comportan trabajo emocional tienen tres características: primero, son trabajos con contacto cara a cara o voz a voz con el público; segundo, producen en otra persona un estado emocional positivo o negativo; y tercero, toleran la supervisión o control de sus actividades emocionales. El control de las emociones del empleado por parte de las organizaciones se realiza con reglas explícitas o implícitas de exhibición emocional (Rafaeli y Sutton, 1987; Diefendorff, Croyle y Gosserand, 2005).

Con respecto a las investigaciones sobre el trabajo emocional en la enseñanza, Tsang sostiene que se han enfocado en contestar dos preguntas de investigación, a saber: "si enseñar incluye trabajo emocional y cuáles serían los efectos del trabajo emocional de enseñar" (2011, p. 1313). Para responder a la primera pregunta, debemos tener en cuenta que el docente sí tiene contacto cara a cara con el público, en este caso, con sus estudiantes y con los padres de familia, y que además usa sus emociones para crear sensaciones tanto en los estudiantes como en sus padres. Hasta aquí tenemos dos de las tres características de las que habla Hochschild para que haya trabajo emocional. Sin embargo, la tercera característica no resulta del todo clara, pues no hay una supervisión directa por parte de la empresa en la creación de dichas emociones en los estudiantes y padres.

No obstante, algunos autores, como Zembylas (2002, 2005, 2003b), han argumentado que existen unas reglas que los docentes deben cumplir con respecto a sus emociones, marcadas por el control organizacional sobre el actuar emocional del profesor. Ye y Chen (2015; citando a Winograd, 2003) dicen que hay por lo menos cinco reglas emocionales en la enseñanza: 1) cuidar a los estudiantes y demostrarles entusiasmo; 2) expresar entusiasmo y pasión por el contenido de la enseñanza; 3) tratar de evitar mostrar emociones extremas en la enseñanza como una exagerada alegría, tristeza o ira; 4) amar su trabajo; 5) tener buen humor para resolver sus propios errores y los errores de los estudiantes. Estas consideraciones fundamentan la labor docente como un trabajo emocional por excelencia.

Dando por sentado que la enseñanza es un trabajo emocional, nos referiremos a la segunda pregunta sobre los efectos del Trabajo Emocional de la Enseñanza (TEE). Tenemos, pues, que este comporta, según diversas investigaciones, efectos positivos (Hargreaves, 2000), pues el acto de enseñar, al ser voluntario, trae consigo satisfacción profesional y laboral, pero igualmente puede comportar efectos negativos en el empleado (Grandey, 2000). Uno de estos es el síndrome de burnout o síndrome de estar quemado, el cual se define como una "respuesta al estrés laboral crónico caracterizada [...] por la sensación de estar agotado a nivel emocional, por el desarrollo de sentimientos negativos, y de actitudes y conductas de cinismo hacia las personas destinatarias del trabajo" (Gil-Monte, 2003, p. 183). Dicho sindrome tiene tres dimensiones: 1) de desgaste emocional, en el cual el empleado se siente crónicamente estresado, agotado y quemado; 2) de despersonalización, de modo que el empleado se distancia de aquellos que son objeto de su trabajo, es decir, de sus clientes, pues considera que es lo mejor para protegerse emocionalmente, lo que lo vuelve frío en el trato interpersonal -algunos autores lo llaman cinismo-; 3) de falta de realización personal en el trabajo, ya que el empleado se siente sin vocación para ejercer sus labores, se cree incompetente y culpable por la despersonalización. Siendo la primer dimensión las más importante, ya que en ella se basan las 
otras dos dimensiones, Kim encuentra que "el burnout está significativamente correlacionado con todas las dimensiones de la labor emocional y que la acción superficial es el más fuerte predictor del desgaste emocional y de la despersonalización" (2016, p. 2). Tenemos entonces que el TEE es uno de los factores para que aparezca el síndrome de burnout en los docentes. Con respecto a la falta de realización personal -tercera dimensión- de los docentes, esta también es parte de todos los aspectos del trabajo emocional (Yilmaz, Altinkurt, Guner y Sen, 2015). Así pues, el trabajo emocional causa efectos positivos y negativos en los empleados, dependiendo de la relación entre las emociones que se muestran y las que realmente se sienten (Özan, 2014; Gracia, Martínez, Salanova y Nogareda, 2007; O'Connor, 2006; Zembylas, 2005).

Como se ve, el concepto de trabajo emocional ha tenido un gran impacto en el estudio y análisis de las emociones en el campo educativo y ha servido de base para desarrollar otros acercamientos al fenómeno emocional de los docentes, como es caso de la geografía de las emociones en la enseñanza.

\section{Geografía emocional de la enseñanza}

La escuela es el escenario por excelencia que posibilita la relación con los otros y, en gran medida, la relación consigo mismo. Es allí donde se hacen más palpables las jerarquías a las cuales se está sujeto y, sin duda alguna, es el lugar donde se forma el concepto de amistad, fundado en la vivencia diaria, pero también es el sitio donde se generan los primeros desencuentros, las primeras guerras, los primeros sentimientos de envidia, de miedo, etc. En definitiva, es el espacio que propicia la socialización y la disociación. Pero esto no solo hace referencia al sujeto estudiante, sino también al sujeto educador, que en su labor diaria y en su relación con los estudiantes, con la estructura escolar, con la pedagogía, con la planeación curricular, con los padres y con los colegas, se moldea no solo intelectual y profesionalmente, sino también a nivel emocional, porque enseñar es una práctica emocional (Hargreaves, 1998, 2000, 2001a/b).

Para describir dicho fenómeno, Hargreaves introdujo un nuevo concepto en la investigación educativa y de las ciencias sociales que denominó geografias emocionales de la enseñanza (GEE), en el cual define "las relaciones de cercanía y distancia que moldean las emociones que sentimos y las relaciones que ayudan a crear, configurar y colorear los sentimientos y emociones que experimentamos con respecto a la relación con nosotros mismos, con los otros y con el mundo que nos rodea" (2001a, p. 1056). El autor inglés - citando a Ashforth y Humphrey (1993)- dice que los profesores, como otros trabajadores del sector de servicios o en la profesión del cuidado, por lo regular invierten un fuerte trabajo emocional o labor emocional para alcanzar una mayor cercanía o distancia de sus clientes (p. 1062).

En este contexto, el autor encontró cinco formas de geografía emocional en la enseñanza que definen la relación entre el docente y los otros miembros de la comunidad educativa:

- Distancias o cercanías socioculturales, relacionadas con la clase, la edad, el género, las sexualidades, la urbanización y otros factores que influyen en los entendimientos que los profesores tienen de su trabajo.

- Distancias o cercanías morales, pues las emociones tienen que ver uno mismo, con las creencias acerca de la educación y con los resultados del propio trabajo, y se confrontan a diario con la realidad del salón de clases.

- Distancias o cercanías profesionales, ligadas al papel que el docente desempeña y debe proyectar, en las que entran en juego la apariencia, la autoridad, el comportamiento, las relaciones con los colegas.

- Distancias o cercanías políticas con los otros, marcadas por las interacciones en la escuela, por el poder que se ejerce y por el estatus que este da. 
- Distancias o cercanías corpóreas de la presencia corpórea, relativas al encuentro en el mismo tiempo y espacio: comprenden aquellas pautas de organización, instalaciones y equipos que influencian los sentimientos de los docentes acerca de su papel en la educación.

Respecto de las geografías emocionales de la enseñanza, Hargreaves (2000) realizó una investigación en donde entrevistó a 60 docentes de primaria y secundaria de 15 escuelas de diferentes niveles y tamaños, que ofrecían sus servicios a diversas comunidades rurales y urbanas. En dicha investigación, el autor encontró que en la interacción docente-estudiante los maestros solían poseer calidez profesional e interactuaban regularmente con sus estudiantes, lo cual mejoró la cercanía física y profesional entre docentes y estudiantes, cercanía que, en cierta medida, era la base para el desarrollo de la comprensión emocional entre profesores y alumnos. Por otro lado, Hargreaves argumentó que "el poder desigual entre estos dos grupos de personas puede conducir a la aversión y rechazo en el ambiente emocionalmente intenso del aula en la escuela primaria" (2000, p. 824). Es decir, que la geografía emocional de la distancia política pone en riesgo el entendimiento emocional dentro del aula, y esto se da cuando los docentes deben hacer uso de su autoridad sobre el estudiante. Es por ello que Hargreaves asevera que "los docentes obtienen muchas de sus recompensas emocionales y construyen relaciones significantes con los estudiantes, por fuera del aula de clase y no en ella" (p. 824). Es otras palabras, las emociones positivas aparecen cuando se minimizan las distancias políticas, que en este caso están enmarcadas en el aula de clase.

Partiendo del marco teórico de las GEE, se han realizado otras investigaciones durante la implementación de reformas curriculares, puesto que "el análisis de las emociones de los profesores mientras se aplican las reformas puede proporcionar una comprensión más profunda de las formas en que los docentes experimentan su trabajo" (Editorial, 2005, p. 895). En dichas investigaciones se ha encontrado que las emociones de los profesores se entrelazan con los procesos de transformación y que ellas influyen en su compromiso con el proyecto de cambio, además que, por el estrés que generan las modificaciones, el profesor pierde la felicidad de comunicarse con los estudiantes (McCaughtry, Martin, Cothran y Hodges-Kulinna, 2006; Yin y Lee, 2006). Así pues, Hargreaves dice que la microgestión de estándares basados en las reformas tiene "efectos nocivos en la enseñanza de los profesores, en los estudiantes de los profesores y en las relaciones de los profesores con su trabajo y con los otros" (2003, p. 90).

\section{Conclusiones}

Las emociones son fenómenos que se escenifican en todos los sitios donde actúan los seres humanos y habitan en todas las relaciones de los sujetos con otros seres humanos, y aun con ellos mismos y con las cosas del mundo; no obstante, en la actualidad su descripción es compleja, ya sea por la manera como se tematizan en la cotidianidad o por la cantidad de literatura y, por tanto, de posturas que dan cuenta de ellas. Respecto de las emociones, encontramos posturas psicológicas, neurológicas, antropológicas, sociológicas y filosóficas que abordan el tema con diferentes fundamentos, por lo que su significación se hace un poco gaseosa; sin embargo, pensar las emociones en general y en relación con la educación nos deja algunas sentencias que podemos enunciar, a modo de cierre de esta revisión de literatura.

Primero, las emociones son estados de ánimo que habitan en cada ser humano de una manera intima, son experiencias individuales, pero se manifiestan en el mundo por medio de expresiones corporales. Si bien cada persona siente las emociones a su manera, existe la forma de reconocer estos estados; es más, ellas se trasmiten de persona a persona como una manera de comunicación. El otro sabe de la tristeza que siente alguien por medio de deter- 
minados gestos que son frecuentes en una persona cuando es invadida por esta emoción.

Segundo, las posturas frente al tema de las emociones son tematizadas desde la época griega, pero han cobrado mucho interés en las últimas décadas, sobre todo en la psicología, la sociología y la neurología. Si bien los enfoques tienen diferentes fines, las bases son similares, pues parten de la concepción de los estados anímicos de los seres humanos.

Tercero, tradicionalmente se pensaba que emoción y razón eran dos dispositivos que tenían que estar separados (Zembyla, 2003a/b; Sutton y Wheatley, 2003). De hecho, se consideraba que aquella podía ser obstáculo de esta, si bien en la actualidad, sobre todo por el lado de la neurología, se ha demostrado que la razón necesita tanto de las emociones como estas de ella.

Cuarto, las emociones juegan un papel fundamental en la construcción social y sobre todo en las interacciones económicas, pues se constituyen en elementos interacciónales definitorios en las negociaciones. En consecuencia, si miramos la educación como una empresa, entonces las emociones, tanto de los maestros como de los estudiantes y padres de familia, son mediadoras en la toma de decisiones en los actos pedagógicos.

Quinto, en esta misma línea, las aulas de clase se constituyen en escenario de las emociones, donde estas juegan un papel primordial en el proceso de enseñanza-aprendizaje, pues de ellas depende la disposición del maestro para enseñar y de los alumnos para aprender.

Sexto, el aula de clase es uno de los espacios primordiales para la interacción emocional y allí se recrea la amistad, el amor, el odio, el resentimiento, entre otras emociones; por decirlo de alguna manera, el aula da lugar a una propedéutica de las emociones.

\section{Referencias}

Ashforth, B. E. y Humphrey, R. H. (1993). Emotional labor in service roles: The influence of identity. Academy of Management Review, 18(1), 88-115.

Camps, V. (2011). El gobierno de la emociones. Barcelona: Herder.

Colhoun, C.y Solomon, R. C. (1989).¿Qué es una emoción? México: Fondo de Cultura Económica.

Damasio, A. (1994). Descartes'Error: Emotion, reason, and the human brain. Nueva York: Putnam [El error de Descartes: la emoción, la razón y el cerebro humano. Barcelona: Crítica, 2006].

Damasio, A. (2005). En busca de Spinoza. Barcelona: Crítica.

Díaz, J. L. y Flores, E. O. (2001). La estructura de la emoción humana: un modelo cromático del sistema afectivo. Salud Mental, 24(4) (agosto).

Diefendorff, J. M., Croyle, M. H.y Gosserand, R. H. (2005). The dimensionality and antecedents of emotional labor strategies. Journal of Vocational Behavior, 66, 339-357.

Editorial (2005). Teaching and Teacher Education, 21, 895-898. 
Ekman, P. (1973). Cross-cultural studies of facial expression. En Darwin and facial expression: A century of research in review (pp169-222). New York Academic Press.

Ekman, P. (1993). Facial expressions and emotions. American Psychologist, 48, 384-392.

Ekman, P. (1998). Introduction. En Ch. Darwin [1872], The expression of emotions in man and animals (pp. xxixxxvi). Nueva York: Oxford University Press.

Ekman, P.y Oster, H. (1981). Expresiones faciales de la emoción. Estudios de Psicología, 7, 116-143.

Freud, S. (1916). Lo inconsciente. En Obras completas (t. 14). Buenos Aires: Amorrortu.

Fried, L. (2011). Teaching teachers about emotion regulation in the classroom. Australian Journal of Teacher Education, 36(3).

Gardner, H. (1997). Arte, mente y cerebro. Una aproximación cognitiva a la creatividad. Buenos Aires: Paidós.

Gazzaniga, M. S. (ed.) (2000). The New Cognitive Neurosciences (2 ed.). MIT Press.

Gazzaniga, M. S. (2008). Human: The science of what makes us unique. Nueva York: Harper Collins.

Gil-Montes, P. (2003). Burnout syndrome: ¿síndrome de quemarse por el trabajo, desgaste profesional, estrés laboral o enfermedad de Tomás? Revista de Psicología del Trabajo y de las Organizaciones, 19(2), 181-197.

Goffman, E. (1981). La presentación de la persona en la vida cotidiana. Buenos Aires: Amorrortu.

Gracia, E., Martínez, I., Salanova, M. y Nogareda, C. (2007). El trabajo emocional: concepto y prevención. Nota Técnica de Prevención 720. Madrid: Instituto Nacional de Seguridad e Higiene en el Trabajo, Ministerio de Trabajo y Asuntos Sociales de España. Recuperado de http://www.insht.es/InshtWeb/Contenidos/Documentacion/FichasTecnicas/NTP/Ficheros/701a750/ntp_720.pdf

Gracia, E., Ramos, J. y Moliner, C. (2014). El trabajo emocional desde una perspectiva clarificadora tras treinta años de investigación. Universitas Psychologica, 13(4), 1517-1529. Recuperado de http://dx.doi.org/10.11144/ Javeriana.UPSY13-4.tepc

Grandey, A. A. (2000). Emotion regulation in the workplace: A new way to conceptualize emotional labor. Journal of Occupational Health Psychology, 5(1), 95-110.

Grandey, A., Diefendorff, J. M. y Rupp, D. (2013). Emotional labor in the $27^{\text {st }}$ century: Diverse perspectives on emotion regulation at work. Nueva York \& Londres: Routledge.

Hargreaves, A. (1998). The emotional practice of teaching. Teaching and Teacher Education, 14(8), 835-854.

Hargreaves, A. (2000). Mixed emotions: Teachers' perceptions of their interactions with students. Teaching and Teacher Education, 16, 811-826. 
ISSN 0123-1294 | e-ISSN 2027-5358 | Educ.Educ. Vol. 20. No. 3 | Septiembre-diciembre de 2017 | pp. 451-465.

Universidad de La Sabana | Facultad de Educación

Hargreaves, A. (2001a). Emotional geographies o teaching. Teachers'College Record, 103(6), 1056-1080.

Hargreaves, A. (2001b). The emotional geographies of teachers' relations with colleagues. International Journal of Educational Research, 35, 503-527.

Hargreaves, A. (2003). Teaching in the knowledge society: Education in the age of insecurity. Philadelphia: Open University Press.

Hochschild, A. R. (1979). Emotion work, feeling rules, and social structure. American Journal of Sociology, 85(3), 551-575.

Hochschild, A. R. (1983). The managed heart: Commercialization of human feeling. Berkeley: University of California Press.

Hochschild, A. R. (1990). Ideology and emotion management: A perspective and path for future research. En T. D. Kemper (ed.), Research agendas in the sociology of emotions (pp. 117-142). Albany: State University of New York Press.

Isenbarger, L. y Zembylas, M. (2006). The emotional labor of caring in teaching. Teaching and Teacher Education, 22, 120-134.

James, W. (1884). What is an emotion? Mind, 9(34), 188-205.

Kim, Y. (2016). Emotional labor and burnout among public middle school teachers in South Korea. Tesis de Maestría, University of Jyväskylä, Finlandía.

Lange, C. G. (1967). The emotions. A psy chophysiological study. En C. G. Lange y W. James, The emotions. Nueva York y Londres: Hafner.

McCaughtry, N., Martin, J. J., Cothran, D. y Hodges-Kulinna, P. H. (2006). The emotional dimensions of urban teacher change. Journal of Teaching in Physical Education, 25(1), 99-119. Recuperado de http://digitalcommons.wayne.edu/coe_khs/19

Morris, J. A. y Feldman, D. C. (1996). Managing emotions in the workplace. Journal of Managerial Issue, 9(3), 257-274.

Nussbaum, M. (2001). Paisajes del pensamiento: la inteligencia de las emociones. Barcelona: Paidós.

O'Connor, K. E. (2008). You choose to care: Teachers, emotions and professional identity. Teaching and Teacher Education, 24, 117-126.

Özan, M. B. (2014). Teaching and emotional labor. American International Journal of Social Science, 3(5).

Plutchik, R. (1980). Emotion: A psy choevolutionary synthesis. Nueva York: Harper \& Row.

Rafaeli, A. y Sutton, R. I. (1987). Expression of emotion as part of the work role. Academy of Management Review, 12, 23-37. 
Sutton R. E. y Wheatley, K. F. (2003). Teachers' Emotions and Teaching: A review of the literature and directions for future research. Educational Psychology Review, 15(4) (diciembre).

Tsang, K. K. (2011). Emotional labor of teaching. Educational Research, 2(8) (agosto), 1312-1316. Recuperado de http://www.interesjournals.org/ER

Vendrell, I. (2009). Teorías analíticas de las emociones: el debate actual y sus precedentes históricos. Universidad Complutense de Madrid.

Ye, M. y Chen, Y. (2015). A literature review on teachers' emotional labor. Creative Education, 6, 2232-2240.

Yilmaz, K., Altinkurt, Y., Guner, M. y Sen, B. (2015). The relationship between teachers' emotional labor and burnout level. Eurasian Journal of Educational Research, 59, 75-90. Recuperado de http://dx.doi.org/10.1468g/ ejer.2015.59.5

Yin, H.y Lee, C. K. J. (2006). Teacher's emotional map in curriculum implementation: A cultural-individual analysis of the Senior Secondary School Curriculum Reform in Guangdong, China. Apera Conference, 28-30 de noviembre, Hong Kong.

Zembylas, M. (2002). Structures of feeling in curriculum and teaching: Theorizing the emotional rules. Educational Theory, 52(2), 187-20.

Zembylas, M. (2003a). Caring for teacher emotion: reflections on teacher self-development. Studies in Philosophy and Education, 22, 103-125, 2003.

Zembylas, M. (2003b). Interrogating "teacher identity": emotion, resistance, and self-formation. Educational Theory, 53(10) (invierno).

Zembylas, M. (2005). Discursive practices, genealogies, and emotional rules: A poststructuralist view on emotion and identity in teaching. Teaching and Teacher Education, 21, 935-948. 
